\title{
Government and Governance of Security. The Politics of Organized crime in Chile
}

\author{
Carlos Solar \\ Routledge Studies in Latin American Politics \\ New York and London, Routledge, Taylor \& Francis Group, 2018 \\ 157 páginas.
}
Como sugiere el título del texto de Carlos Solar, uno de los te- mas de mayor relevancia y preocupación en las agendas de las políticas públicas y en los estudios especializados sobre institucio- nalización y participación en sociedades democráticas contempo- ráneas, es el gobierno y la gobernanza de la seguridad en el campo del crimen organizado.

En efecto, a partir de un estudio de caso, el trabajo aborda las amenazas a la seguridad y capacidades de respuesta de las instituciones gubernamentales nacionales en Chile, a partir del proceso de retorno a la democracia, esto es, desde los inicios de 1990 hasta mediados de la presente década. Para los fines explicativos del objeto de estudio, de qué manera la evolución del proceso de redemocratización ha afectado las formas de gobernanza en seguridad.

Ciertamente que la selección de período de transición política y democratización reciente de la sociedad chilena, ofrece un punto de inicio propicio para analizar y evaluar el desarrollo y dinamismo de políticas y estrategias institucionales y gubernamentales respecto del tráfico de drogas, lavado de dinero y contrabando humano, como de sus efectos y amenazas crecientes para la naciente democracia sudamericana.

En la primera parte del libro y a modo de introducción, su autor identifica y dimensiona la naturaleza y alcances de la seguridad y crimen organizado como retos crecientes para el estado en nuestros días. Asimismo, expone y justifica con fundamentos, la selección del caso chileno post autoritario para su estudio, puesto que lo califica como expresión de países que se han visto enfrentados a esta 
situación demandante y compleja y han podido responder a través de políticas incrementales y parciales a esta nueva realidad, a través del crecimiento y desarrollo de una comunidad policial criminal diversa y más transversal y consensual en sus criterios funcionales.

Adicionalmente, uno de los argumentos que se esgrimen para la selección de Chile democrático como caso de estudio en el ámbito de su exposición al crimen transnacional, es su comparación en el concierto latinoamericano. A saber, estabilidad política, crecimiento de mercados financieros-comerciales y estándares de consumo. Asimismo, las tasas de criminalidad, victimización de corrupción y percepciones frente al crimen organizado, permanecen entre las más bajas de la región. Por último, la dimensión demográfica y la naturaleza centralizada del Estado ameritan estudiar el caso chileno, por cuanto permite un mejor manejo de la administración de la justicia criminal en comparación con gobiernos de naturaleza federal existentes en la región latinoamericana.

Desde un punto de vista de los enfoques conceptuales introducidos por el autor para la comprensión y análisis del caso de estudio, estos se explican a través de los postulados de la gobernanza, redes políticas e institucionalismo histórico. La combinación de estas tres perspectivas y su utilización individual permiten una base teórica para la lectura e interpretación del crimen organizado en Chile.

De este modo, acuñando la conceptualización de gobernanza (estudio de formaciones, experiencias y respuestas a los dilemas de gobierno), más sus procesos de medios y capacidades por lograr bienes colectivos como resultados de la interacción crecientemente horizontal de diversos actores, la propuesta empírica del estudio apuesta afirmar que la gestión gubernamental chilena de los temas criminales sugiere que los actores políticos, judiciales y económicos, entre otros, están constantemente forzados a proveer políticas colaborativas para confrontar el tráfico de drogas, lavado de dinero, corrupción y mercados negros.

En consecuencia, bajo la observación teórica, el autor del libro postula que aunque la gobernanza del crimen organizado ocurre bajo la autoridad del Estado, la multiplicidad de miembros involucrados en esta, ha roto los estrictos proce- 
sos jerárquicos que enfatiza la literatura, formando - por el contrario- interacciones horizontales con propósitos determinados. A esta moción la define como el movimiento desde el gobierno a la gobernanza.

En síntesis, el capítulo introductorio del libro anticipa los objetivos más importantes del estudio, destacando, entre ellos, la reciente necesidad de incorporar a los análisis del crimen organizado las tendencias de gobernanza del mismo en Chile. Ofrece también la oportunidad de explorar nuevos argumentos teóricos, tomando en consideración el carácter diferente del caso chileno en la región latinoamericana y en el mundo en desarrollo. En este sentido, la propuesta de analizar las políticas para combatir el crimen organizado y llevadas adelante en forma corporativa o transversal por instituciones como el Ministerio del Interior, cuerpos políticos, el Ministerio Público, el Consejo de Defensa del Estado, la Unidad de Análisis Financiero y las Cortes de Justicia, pueden ser muestra de un proceso de evolución de gobiernos democráticos que se comprometen y comienzan a actuar en los escenarios de gobernanza de la seguridad moderna. Sin perjuicio de la especificidad de la situación chilena, la verificación de una gobernanza democrática en términos de pautas, formaciones, experiencias y respuestas a los dilemas gobernantes, en vez de indicadores de resultados, como explora este estudio, podría extrapolarse a otros casos similares en la región, como por ejemplo, Uruguay y Costa Rica.

La fundamentación teórica del paradigma de la gobernanza del crimen organizado se explaya e ilustra en el segundo capítulo del texto. Los enfoques y debates centralizados y descentralizados sobre el rol del Estado explican el movimiento desde el gobierno a la gobernanza en la construcción de la seguridad. Por qué la gobernanza, qué tipo de gobernanza, jerárquica o desde abajo, para quién y cómo se desarrolla en diferentes contextos políticos, culturales y regionales. De igual forma se sustenta la argumentación conceptual en términos de la utilidad y funcionalidad de las redes políticas en la explicación de expresiones de gobernanza del crimen organizado, en especial su aplicabilidad para el caso de las instituciones chilenas. La evolución, cambio y adaptación que estas experimentan en el tiempo se explican conceptualmente a través 
del institucionalismo histórico. Por lo tanto, una pregunta central nos remite a la relevancia de las estructuras democráticas, es decir, hasta qué punto la formación institucional y reformas de las mismas en el proceso de redemocratización chilena ofrecen respuestas a la gobernanza gradual del crimen organizado.

Interrogantes y argumentaciones como las que se plantean en la discusión conceptual son puestas a prueba a través de una propuesta metodológica de herramientas cualitativas, útiles en el examen de procesos de gobernanza en el tiempo. Sobre la base de una estrategia de colección de documentos escritos, la metodología empleada en el libro se centra en la revisión de documentos históricos y contemporáneos, como de datos orales por medio de entrevistas exploratorias. Más específica y empíricamente, se recurre a una estrategia de entrevistas de élite con el objeto de explicar el significado y autenticidad de las acciones y decisiones adoptadas por personeros representativos de diversas reparticiones públicas seleccionadas para el estudio, como así también representantes del sector privado y de la sociedad civil, que tengan relación con las políticas de gobernanza del crimen organizado en Chile. En los capítulos siguientes, desde el tercero al quinto, el texto se concentra primeramente en una breve narración histórica y caracterización de los rasgos centrales y políticas de abordaje del crimen organizado en Chile, bajo el régimen militar, tras el golpe de Estado de 1973 y el retorno a la democracia en 1990. No obstante, es a los inicios del proceso de reconstrucción democrática, el contexto a partir del cual el estudio focaliza el análisis empírico de fuentes documentales y entrevistas para la explicación acerca de la evolución que representa en forma dinámica y compleja la interacción entre crimen organizado e intentos de gobernanza del mismo. Los cambios experimentados por ambas variables durante el transcurso de los gobiernos civiles democráticos hasta mediados de la presente década, se reflejan en la constatación y verificación de un tipo de estrategia de políticas públicas que evoluciona y se adapta frente a la complejidad de los desafíos y amenazas de la seguridad que enfrenta la gobernanza democrática y la sociedad civil chilena. 
Como manifestaciones de estas adecuaciones institucionales y gobernanza del crimen organizado, los capítulos correspondientes dan cuenta de la estructuración y funcionamiento de redes interinstitucionales con la creación y participación determinante de instancias como el Ministerio Público y la Unidad de Análisis Financiero, a partir del año 2000 en adelante. Así es posible verificar las observaciones y planteamientos iniciales del autor en cuanto a una mayor horizontalidad y reemplazo en la dirección de la gobernanza del crimen organizado por parte del gobierno y de la policía. Para los efectos de alcance político, se explora también el grado en que las redes de seguridad en formación se sustentan en una cultura de responsabilidad democrática emergente por parte de todos los actores de la sociedad y de desempeño institucional basados en acuerdos mutuos sobre la materia.

En línea con lo anterior y conforme con los argumentos teóricos del estudio, el capítulo quinto da cuenta de una nueva estrategia nacional de seguridad y gobernanza del crimen organizado, en el campo de la seguridad financiera y adoptada en 2012 por los ministerios del Interior y Hacienda. En efecto, la Estrategia Nacional contra el Lavado de Dinero y Financiamiento Terrorista (ENLAFT) consiste en una alianza estratégica de colaboración interinstitucional entre ambos ministerios con la Unidad de Análisis Financiero (UAF). Esta alianza es producto también de una colaboración entre instituciones públicas y privadas con asesoría internacional del Fondo Monetario Internacional (FMI) y Banco Interamericano (BID). Esta nueva estrategia marca cambios significativos de dirección en la gobernanza contra el crimen organizado, desde el punto de vista del liderazgo de nuevas instituciones en los procesos de gobernanza, como así también en la mayor horizontalidad en la participación interinstitucional como de actores de la sociedad civil, dando origen a redes más amplias e inclusivas.

Del mismo modo, el posicionamiento y liderazgo del nuevo Ministerio Público a partir de $\mathbf{2 0 0 4}$, en la labor de fiscalía y procesamiento judicial, es decisivo y complementario respecto del equilibrio alcanzado con el brazo político del $\mathrm{Mi}$ nisterio del Interior en la gobernanza del crimen organizado. 
Una muestra de esta interacción es la participación agregada de la Fiscalía en las mesas redondas y en los intercambios bilaterales de rutinas y protocolos con otras dependencias de las redes interinstitucionales, y en los encuentros y reuniones intersectoriales que, más tarde, dan origen a planes de políticas en seguridad.

El capítulo sexto concluye con una reconstrucción teórica en términos de una comprensión acerca de la naturaleza y gobierno de los procesos alrededor de los temas de seguridad. Junto con analizar las condiciones y circunstancias para la construcción de redes de gobierno, se evalúan los factores que obstaculizan las relaciones interinstitucionales y cuáles son las consecuencias para la gobernanza democrática bajo un desarrollo institucional constante en el campo de la seguridad. La evolución y cronología de la gobernanza de la seguridad en crimen organizado en Chile se resume también en este capítulo, para dar cuenta de los diferentes estadios y cambios observables conforme a los supuestos y exploraciones del autor sobre la materia.

Finalmente, el texto concluye con recomendaciones de políticas, en particular atendiendo las preocupaciones y demandas de los profesionales y decidores de políticas públicas. Entre ellas destacan que la mejor manera de enfrentar el crimen es a través del reconocimiento, por parte de los actores en el proceso decisional, del carácter horizontal, multi jurisdiccional e inter institucional de su gobierno. Asimismo, el proceso debe incluir una participación amplia de actores envueltos en la materia de seguridad, incorporando actores tanto nacionales como internacionales en las negociaciones entre las partes para los fines de buscar cohesión y consistencia en los resultados de las políticas.

La gobernanza también debe incorporar actores fuera del Estado, siempre de acuerdo a su participación en redes de seguridad que deberían estar alineadas con reglas democráticas que se asocie a un sistema integrado de valores. En suma, sobre la base de la experiencia chilena, las recomendaciones incluyen una serie de condiciones y pasos para la gobernanza del crimen organizado. 
A modo de conclusión, el caso de estudio que presenta el libro de Carlos Solar constituye un aporte conceptualmente sólido y metodológicamente coherente con los argumentos y verificación empírica, conforme a los planteamientos y fundamentos del libro. Representa, asimismo, una sugerente reflexión y propuesta para la investigación científica, como así también para la recomendación de políticas públicas internacionales en una materia de gran relevancia e interés, tanto en el mundo académico y especializado, como para los gobiernos y actores políticos comprometidos con la gobernanza de la seguridad en el contexto contemporáneo.

\section{POR José A. Morandé L.}

Instituto de Estudios Internacionales

Universidad de Chile 
